\title{
Les usages numériques des lycéens affectent-ils leur temps de travail personnel?
}

\author{
François Burban, Philippe Cottier, Christophe Michaut (CREN, Nantes)
}

RÉSUMÉ • 1618 lycéens ont été interrogés sur leurs pratiques numériques, leurs manières de travailler et le temps qu'ils y consacrent. Les résultats de cette recherche mettent en évidence le rôle pondéré joué par les pratiques numériques des lycéens sur la variation du temps de travail personnel en regard de caractéristiques sociodémographiques, du diplôme préparé et des manières d'étudier.

MOTS-CLÉS • Lycéens, temps de travail, usages du numérique.

ABSTRACT 1618 high school students were interviewed on their digital practices, their way to work and how much time they spend using them. The results of this survey brings out the weighted role performed by digital practices of these students, their homework time according to their socio-demographic features, the diploma prepared and their way to study.

KEYWORDS • high school students, homework time, digital practices. 


\section{Introduction}

Dans un monde fortement marqué par le numérique, les interrogations, voire les affirmations concernant les pratiques des jeunes et leur influence sur leur travail scolaire sont nombreuses. Ces discours, tantôt produits par les parents, tantôt par les institutions, les enseignants ou les politiques, stigmatisent tour à tour les bénéfices et les risques des usages de l'ordinateur chez les adolescents. Certains voient dans le numérique le développement de nouvelles compétences tandis que d'autres craignent que les activités numériques ne se substituent aux activités scolaires. L'enchevêtrement des pratiques ludiques et éducatives est manifeste: navigation sérendipitaire, gestion de réseaux sociaux, jeux en ligne, consultation de ressources vidéos, communication par SMS ${ }^{1}$, utilisation de l'internet pour faire ses devoirs, échange de corrigés ou de cours, etc. Dans quelle mesure ces différentes activités affectent le temps de travail scolaire des lycéens?

Évaluer les effets des technologies numériques est une demande sociale qui est récurrente. Chaque technologie nouvelle, en particulier dans le domaine de l'éducation, s'accompagne de discours apologétiques sur les vertus intrinsèques qu'elles seraient sensées porter (Cuban, 1986), une «ambiguïté tragique » débute à chaque nouvelle invention : les technologies résultent de processus industriels et sont diffusées alors que les applications sociales restent à inventer (Dieuzeide, 1982) ; une nouvelle idéologie, porteuse de mythes communicationnels, s'est installée (Breton \& Proulx, 1989) (Mattelart \& Mattelart, 2004) (Mattelart, 1994). L'offre technique et les discours qui l'accompagnent ne se traduisent pourtant pas nécessairement, loin de là, par des usages qui sont conformes aux espérances.

Il est difficile, comme le montre l'évolution des recherches dans le domaine des technologies de l'information et de la communication (Mattelart \& Mattelart, 2004), de répondre à la question de l'impact des dispositifs techniques en matière de communication. La complexité des situations étudiées appelle nécessairement la prise en compte, mesurée et

${ }^{1}$ Short Message Service. Nous utilisons indifféremment dans cet article les termes « texto » ou «SMS » 
pondérée, de variables contextuelles au sein desquelles les pratiques des TIC $^{2}$ ne jouent qu'un rôle parmi d'autres. La causalité entre situation étudiée et pratiques numériques se dissout en effet dans un entremêlement de variables qui agissent les unes sur les autres dans un système souvent difficile à circonscrire.

Tout projet d'évaluation des effets se heurte donc à un écueil méthodologique. Comment rendre compte des impacts potentiels de technologies de l'information et de la communication? La sociologie des usages propose d'y répondre par une inversion des pôles, en posant la question des pratiques des usagers plus que celle des effets qui les affecteraient, s'intéressant à la production, à l'émergence dans un contexte donné de pratiques plutôt que tenter de mesurer les effets de tel ou tel dispositif technique. Les résultats de ces travaux, menés depuis près de 40 années en France (Jouët, 2000) (Jouët 2011), ont ainsi montré le jeu qui s'installe entre prescriptions technologiques et dimensions sociales dans une médiation entre technique et social.

Les recherches sur les usages des technologies de l'information et de la communication dans le domaine de l'éducation sont nombreuses, portant sur les pratiques des outils numériques des enseignants ou des élèves dédiés à l'enseignement, sur les équipements ou bien encore sur les programmes de dissémination de technologies numériques dans les établissements. Elles s'attachent principalement à en comprendre et en évaluer les usages mais laissent bien souvent dans l'ombre les interactions entre "nouvelles » technologies et outils plus « anciens » ou plus «traditionnels». Il en va de même pour les pratiques des acteurs qui ne sont fréquemment révélées qu'à l'aune des systèmes numériques étudiés, au risque d'une surdétermination du rôle de la technique. D'autres s'attachent à comprendre le jeu entre compétences mises en œuvre par les jeunes dans leur sphère privée et compétences scolaires. En revanche, les travaux qui s'intéressent à la formation des pratiques des individus en milieu scolaire et tentent de comprendre et mettre à jour la part de l'influence des systèmes techniques en regard d'autres facteurs sont moins nombreux. Partant de ce constat, la recherche dont il est ici question, plutôt qu'étudier ce que produisent les lycéens avec les outils numériques, tente de comprendre ce qui fait fluctuer leur temps de travail personnel.

\footnotetext{
${ }^{2}$ Technologies de l'information et de la communication.
} 
Il convient de rappeler que ce temps consacré aux devoirs et aux leçons est très variable. Il peut résulter de trois causes : l'efficacité dans la production (certains élèves plus efficaces que d'autres consacreront moins de temps), la «méticulosité » de la production qui entraine une augmentation de la consommation du temps (e.g. mettre sa prise de note au propre) et l'inégale exigence du temps prescrit (quantité de devoirs) par l'institution scolaire selon la classe fréquentée par l'élève (Perrot, 1984).

Le numérique intervient sur chacune de ces causes: en rendant la production plus efficace (faire des calculs statistiques à partir d'un logiciel de traitement de données, accéder à une information, traduire, obtenir une définition, récupérer un corrigé, etc.), en permettant de "soigner » la production (taper ses cours sur ordinateur), en prescrivant plus ou moins de compléments au cours (supports numériques, liens vers des sites internet, etc.). Toutefois, la prise en compte du numérique nécessite d'inclure des usages dont la finalité n'est pas exclusivement ou peu scolaire. Certains usages peuvent en effet affecter l'efficacité du temps de travail personnel (papillonnage sur Internet, communication sur les réseaux sociaux, etc.).

Dans quelle mesure l'utilisation des outils numériques affecte-t-elle le travail personnel des lycéens? Nous ferons l'hypothèse que la fréquence d'utilisation du numérique est corrélée au temps de travail personnel. Plus précisément, nous supposerons que le temps et la régularité du travail personnel diminuent avec la fréquence d'utilisation du numérique « ludique » (réseaux sociaux, jeux sur Internet, etc.) et augmente avec le numérique «scolaire » (logiciels bureautiques).

Afin de traiter cette question, un questionnaire a été diffusé entre octobre et décembre 2012 à un échantillon d'élèves inscrits dans l'un des 298 lycées (général, technologique et professionnel) d'une académie. Sa diffusion s'est faite dans une salle informatique des établissements sollicités qui étaient, en outre, invités à faire remplir le questionnaire en garantissant l'anonymat des personnes, dans une classe de seconde, une classe de première et une classe de terminale.

Le questionnaire comporte les thèmes suivants : outils et pratiques numériques à l'école ; scolarité antérieure ; formation suivie ; manières d'étudier ; possession, fréquence et type d'utilisation d'outils numériques en dehors de l'école ; caractéristiques sociodémographiques ; activités de loisirs. 
Finalement, 52 établissements ont accepté de diffuser le questionnaire et 1618 lycéens ont répondu à l'intégralité des questions. Les établissements n'ayant pas rigoureusement respecté les consignes énoncées dans le protocole de recherche, un redressement des résultats bruts a été nécessaire de manière à produire des statistiques valides. Le redressement a été effectué selon le genre, la spécialité de formation (générale, technologique, professionnelle) et le statut (public ou privé) de l'établissement. La qualité de l'échantillon ainsi redressé a ensuite été testée par l'intermédiaire de l'origine sociale des élèves et leur niveau d'études (seconde, première, terminale). Cet échantillon présente des caractéristiques qui ne sont pas significativement différentes de celles de la population académique. Tous les niveaux de formation et toutes les disciplines sont suffisamment représentés pour apprécier le temps consacré aux devoirs et les temps de pratique numérique scolaire et ludique des jeunes.

Deux biais possibles doivent néanmoins être évoqués. Dans la mesure où les résultats sont obtenus sur la base d'une auto-déclaration et non d'une observation, un biais de désirabilité sociale n'est pas à exclure. Par ailleurs, l'imbrication des temps passés à certaines activités peut compliquer leur évaluation, par exemple, le temps consacré aux devoir tout en effectuant des recherches sur Internet et en communiquant sur un réseau social ou par SMS.

Cet article se compose de deux parties. La première est consacrée à l'économie des activités lycéennes. Elle permet d'identifier la place qu'occupent les pratiques numériques et de déterminer quelques dimensions essentielles du travail des lycéens. La seconde, qui s'appuie sur les résultats de l'enquête, décrit tout d'abord le temps de travail personnel des lycéens et celui mobilisé par les activités numériques. Puis, grâce à une analyse multivariée, la variance du temps de travail personnel est rapportée aux caractéristiques individuelles des lycéens, à leur scolarité et à leurs pratiques scolaires et numériques. Nous pouvons ainsi dégager l'effet, toutes choses égales par ailleurs, de certaines pratiques numériques sur le temps de travail personnel des lycéens.

\section{Les activités lycéennes: une économie entre pratiques électives, ludiques et travail scolaire.}

Le temps consacré au travail scolaire personnel dépend de nombreux facteurs liés aux prescriptions enseignantes, aux manières de faire des 
jeunes, à leur environnement familial, etc., mais est aussi fortement marqué par des pratiques électives numériques qui caractérisent fortement l'adolescence.

\subsection{Adolescence et pratiques numériques}

Dans les sociétés démocratiques contemporaines un statut spécifique de la jeunesse s'est progressivement installé, suivant en cela une évolution historique déjà marquée par l'émergence antérieure d'un statut de l'enfant. La reconnaissance de cet âge de l'individu accompagne celle d'une culture qui tend à se distinguer des autres âges de la vie. Cette culture adolescente se caractérise par un certain nombre de dimensions que la sociologie de la jeunesse a identifié, notamment une tendance à vouloir se distinguer des autres catégories d'âge : une forme de défiance envers le monde des adultes, la revendication à l'autonomie, des codes et un langage propres à ce groupe d'âge.

La large diffusion des technologies de l'information et de la communication et les usages qu'elles permettent imprègnent cette culture adolescente plus encore que les autres catégories de la population. Ce fossé générationnel entre les jeunes et leurs ainés a abondamment été commenté. Prensky, s'appuyant sur une métaphore de l'apprentissage de la langue, oppose ainsi «digital natives » et «digital immigrants», les natifs (nés avec le numérique et s'étant alphabétisés très tôt) et les immigrants dont les pratiques plus laborieuses se caractériseraient par un certain «accent » : "Today's older folk were "socialized" differently from their kids, and are now in the process of learning a new language » (Prensky, 2001). Bovill et Linvingstone (Bovill \& Livingstone, 2001) puis Glévarec (Glevarec, 2010) ont montré combien la chambre de l'enfant devenait, grâce aux terminaux d'information et de communication, un nouvel espace de socialisation, évoquant une «culture de la chambre ». Dominique Pasquier insiste elle aussi sur une sociabilité juvénile marquée par une certaine autonomie relationnelle, extra familiale, que les adolescents peuvent développer grâce aux technologies de la communication et sur la possibilité d'ouverture à des mondes extérieurs à leur environnement proche (Pasquier, 2005).

Cette dimension nouvelle marque significativement les pratiques des jeunes et le temps qu'ils consacrent aux activités numériques : communication, sociabilité, activités ludiques. Pour Mercklé et Octobre: 
"L'adolescence se caractérise par une réorganisation des agendas culturels, qui prend une double forme: celle d'une redéfinition des répertoires de loisirs, et celle d'une modification des rythmes de chaque activité » (Mercklé \& Octobre, 2012). On assiste ainsi à une démultiplication des activités et des engagements dans des temporalités proches ou entremêlées, fortement liées au numérique. Dans un portefeuille global d'activités, la part prise par le numérique entraîne la mise en place de «temps partagés » dans lesquels les activités ne s'inscrivent plus dans des temporalités différenciées mais plutôt dans des agencements plus ou moins stables par lesquels le jeune tente d'articuler ses attentes de réalisation personnelle avec certains impératifs extérieurs (relations en famille, communication avec les pairs, travail scolaire, etc.). Les technologies constituées de terminaux (ordinateurs et Smartphones) associées à de nombreuses applications logicielles et à l'internet, concentrent, en même temps qu'elles les rendent polyvalentes, ces pratiques communicationnelles et informationnelles, tant sur le plan de la sociabilité juvénile que du travail scolaire.

Avec l'avancée dans l'adolescence, le numérique entraîne des changements dans le rapport aux savoirs des lycéens, ses manières de travailler la matière scolaire. Selon Proulx, le web et son association avec la microinformatique procure un cadre quasi naturel pour les jeunes qui y trouvent une source privilégiée d'information, pour des besoins personnels comme scolaires (Proulx, 2004). L'usage des moteurs de recherche est très familier pour les lycéens et conviendrait davantage à leur mode de vie qu'une bibliothèque physique ou en ligne (OCLC, 2005). Les usages des moteurs de recherche et de Wikipédia par exemple empiètent sensiblement sur le territoire traditionnel des recherches documentaires en bibliothèque (Donnat, 2009).

Entre pratiques électives et travail scolaire, les lycéens développent des stratégies qui leur permettent d'organiser et articuler temps de loisirs et temps de travail personnel scolaire. La pratique documentaire en ligne y occuperait une place importante. Selon une enquête qualitative menée auprès de lycéens de terminale (Delaunay-Téterel \& Le Douarin, 2011) quatre régimes temporels élaborés au croisement de la fréquence des sorties des lycéens et des types de loisirs qu'ils pratiquent, peuvent être distingués : «la pluriactivité », « une année casanière », « l'optimisation » et «une année de labeur ». Trois logiques d'usages sont identifiées. La première, instrumentale, vise à se mettre en règle avec l'institution scolaire ; la deuxième a pour but de compléter et renforcer les cours, à réviser ; la troisième cherche à compenser les carences de certains enseignements. 
Chaque lycéen, quel que soit son régime temporel, adopterait tour à tour ces trois stratégies. En particulier, en réponse à un système de forte pression scolaire, les lycéens développeraient une stratégie instrumentaliste, plus tournée vers les résultats scolaires que le travail intellectuel et trouveraient en internet le moyen de se développer : «ils puiseraient, dans le web scolaire, des éléments pour tenir et faire face aux exigences scolaires et aux sollicitations multiples ». Mercklé et Octobre montrent par leur étude longitudinale que la pression scolaire s'accroissant, du collège au lycée, la recherche sur internet devient chez les lycéens une pratique qui se renforce avec le temps (Mercklé \& Octobre, 2012).

Pour autant, au-delà de l'apparente homogénéité des pratiques et de l'importance du fait générationnel décrits par bon nombre de travaux, de fortes disparités subsistent dans les pratiques des adolescents. La démocratisation de l'accès aux terminaux et ressources ne suppose pas une absence de stratification des usages connectés. Il existerait ainsi des différences significatives selon les origines sociales et le genre, fruits d'influences parentales qui seraient moins affaire de transmissions explicites de pratiques que d'imprégnation de "ressources dispositionnelles génériques (par exemple, des rapports à l'intimité et à l'exposition de soi) [...]» (Mercklé \& Octobre, 2012) .

Ce processus de transmission générationnelle implicite, associé à des différences d'équipements, se traduit par une plus grande mixité des pratiques chez les adolescents de milieux favorisés (utilitaires ou communicationnelles et scolaires) qu'issus de familles populaires dont les pratiques sont plus récréatives. Selon les milieux sociaux, l'usage qu'en ont les parents - entre utilitaire et ludique - apparaît comme formateur de ceux de leurs enfants. On relève ainsi une forme "d'éclectisme numérique » des adolescents favorisés, éclectisme «que la sociologie de la culture regarde depuis une vingtaine d'années comme la nouvelle marque de fabrique de la «distinction» (Mercklé \& Octobre, 2012). On retrouve ici la récurrence des débats sur la massification du système scolaire et le déplacement des stratégies distinctives, différemment interprétées et mises en œuvre selon les milieux sociaux d'appartenance. La reproduction, voire le renforcement des inégalités scolaires s'incarnerait alors dans des pratiques numériques plus précocement diversifiées chez les jeunes de milieux favorisés que de milieux populaires.

Enfin, les usages des lycéens en matière de numérique varient aussi fortement entre les garçons et les filles. Mais ces différences sont faibles, 
voire inexistantes, à l'entrée dans l'adolescence où l'utilisation de la messagerie, comme de l'ordinateur, ne semblent pas être clivants : garçons et filles en seraient tout autant utilisateurs. C'est essentiellement ce qu'ils en font plus tard qui les distingue, les garçons développant dans le temps des usages plus liés aux loisirs que les filles qui associent plus volontiers finalités scolaires et divertissement: "aux filles des usages plutôt scolaires, communicationnels et créatifs, aux garçons les usages récréatifs et techniques » (Mercklé \& Octobre, 2012).

\subsection{Qu'est-ce que le temps de travail scolaire ?}

La définition et la mesure du temps de travail des élèves est un problème courant dans la littérature scientifique. Dans leur typologie du temps scolaire, adossée aux travaux antérieurs de Crahay (Crahay, 2012), Meuret et Bonnard distinguent le travail «en classe » et le travail « hors classe » (Meuret \& Bonnard, 2010).

Le temps « en classe » articule plusieurs dimensions : «temps prescrit » défini par les programmes officiels, correspondant au temps de présence de l'enseignant en classe que le système scolaire s'est engagé à offrir à l'élève ; le "temps de travail », ou "temps alloué » (dénommé par Meuret et Bonnard comme «temps pris par l'école») où l'élève est en classe et a donc renoncé à consacrer son temps à autre chose ; et l'«effort », temps où l'élève est vraiment engagé en classe dans une tâche scolaire (être attentif à la leçon ou faire un exercice).

Le travail « hors de la classe », qui nous intéresse ici, comprend le soutien scolaire ou les études dirigées, le travail à la maison (ou à l'internat) demandé par l'école, les cours particuliers. Il peut comprendre le temps prescrit non effectué comme par exemple celui résultant des absences d'enseignants. De ce temps de travail il faut soustraire, comme pour le travail en classe, le temps d'installation. Ce travail en dehors de la classe comprend l'« effort hors classe ", c'est-à-dire le temps où l'élève est effectivement engagé dans une tâche scolaire hors de la classe puisque ce temps de travail personnel peut être entrecoupé d'activités non scolaires comme rêvasser, écrire des textos, consulter sa messagerie, flâner sur Internet, etc. Le temps consacré à cette activité n'est pas négligeable si l'on considère qu'au début des années 90' les collégiens français déclaraient consacrer en moyenne 7H45 par semaine au travail à domicile pour l'école (Grisay, 1997). L'estimation de l'ensemble du travail réalisé par les 
lycéens en dehors de l'école ou même en dehors du temps de cours (Barrère, 1997) reste cependant peu aisée à appréhender dans la mesure où il s'avère méthodologiquement difficile d'en observer toutes les composantes autrement que de façon déclarative.

Du point de vue des apprentissages ou des évaluations scolaires, l'investissement dans un ensemble diversifié de tâches peut s'avérer rentable comme contreproductif. Sur ce point, les travaux portant sur la rentabilité des investissements scolaires des élèves montrent l'existence d'un effet de seuil à l'école primaire. L'utilité du travail semble perdre de sa pertinence lors du dépassement d'une certaine quantité (Suchaut, 2009). Quelles que soient les conditions de l'enseignement, un élève dont les aptitudes sont fortes aura potentiellement besoin de moins de temps que celui qui lui est accordé pour réaliser son travail (Carroll, 1963).

Dans l'évolution de son parcours scolaire, le lycéen intègre une technicisation progressive dans la réalisation des tâches scolaires elle-même liée à un mouvement global de technicisation dans le système éducatif. Pour Anne Barrère, cette technicisation se traduit par une décomposition des savoir transmis et de leur restitution en un ensemble de tâches de plus en plus techniques, dont l'objectif n'est plus tant de restituer un savoir que d'acquérir un savoir-faire ou, pour reprendre une expression largement diffusée, d'apprendre à apprendre (Barrère, 2011). La technicisation du travail impacte implicitement toute les étapes du travail et corrélativement sa rentabilité, de la réception du cours en classe, aux devoirs réalisés à la maison et passant par la prise de notes, l'apprentissage et la maîtrise du cours ou la recherche d'informations complémentaires.

Enfin, les lycéens ont parfois des difficultés à gérer globalement leur temps en raison d'une double journée de travail qui résulte de l'ensemble des tâches précédemment citées restant à réaliser en dehors du temps des cours. La pression consécutive à cette «externalisation » des devoirs scolaires (Rayou, 2009) les mène à trouver un équilibre, voire à définir un ordre de priorités dans leurs travaux scolaires et dans un ensemble plus global d'activités sportives, culturelles, relationnelles, etc. Dans cette économie des activités lycéennes, les pratiques numériques des adolescents occupent une place importante.

\section{L'impact du numérique sur le travail personnel des lycéens}


Combien de temps les lycéens accordent-ils au travail scolaire personnel, en dehors des cours et aux pratiques numériques communicationnelles, ludiques et scolaires ? Ces pratiques numériques, en regard d'autres variables explicatives, affectent-elles le temps de travail personnel des lycéens et dans quelles proportions?

L'étude quantitative de notre corpus permet de déterminer combien de temps consacrent les élèves aux activités scolaires hors de la classe activités réalisées dans la sphère domestique mais également celles qui se font au sein des établissements scolaires (salle d'études, CDI, Internat...), dans les lieux publics (bibliothèques) ou privés (domicile des amis) - et les durées qu'ils consacrent aux activités numériques.

Elle permet aussi d'expliquer la disparité du temps de travail personnel en fonction des caractéristiques sociodémographiques des lycéens, du diplôme qu'ils préparent, de leurs manières d'étudier et de leurs pratiques numériques.

\subsection{Durée du travail personnel et temps numériques}

À la question: «En moyenne, combien de temps travaillez-vous chaque jour en dehors des cours (à votre domicile, au CDI, en permanence, etc.) ?», la majorité des lycéens déclare travailler moins d'une heure par jour.

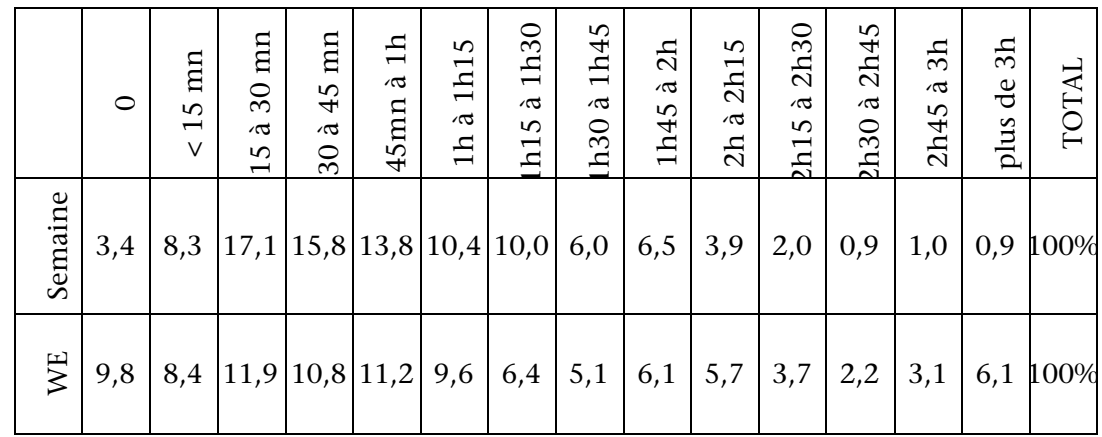

Tableau 1 - Distribution du temps de travail scolaire quotidien hors classe (en \%). 
La durée hebdomadaire moyenne est de 7h13 et l'écart-type de 4 h5 $53^{3}$. Ce dernier résultat et le tableau précédent révèlent une forte disparité dans le temps consacré aux devoirs et aux leçons.

Par ailleurs, $65,1 \%$ disent travailler tous les jours ou presque tous les jours, $18,7 \%$ irrégulièrement et 16,2\% uniquement à l'approche d'une épreuve ou d'un devoir à rendre. Si l'on se réfère aux travaux de Galland et Garrigues (Garrigues \& Galland, 1989), le temps de travail a diminué depuis 25 ans. En 1989, les lycéens travaillaient $68 \mathrm{mn}$ par jour et les lycéennes $88 \mathrm{mn}$. Désormais, ils travaillent respectivement $59 \mathrm{mn}$ et 70 mn selon notre enquête.

Le temps consacré aux activités numériques apparait quant à lui bien plus conséquent. Le tableau suivant (Cf. Tableau 2) révèle que les lycéens sont des usagers patentés d'appareils et d'outils numériques. En moyenne, ils passent beaucoup de temps à communiquer par SMS (2h25/jour) ou sur des réseaux sociaux (1h11), à visionner des vidéos ou écouter de la musique sur Internet (1h05), à jouer sur un ordinateur, un téléphone ou une console (1h05) et à chercher des informations sur un moteur de recherche $(47 \mathrm{mn})^{4}$. Mais, là encore, il existe une très forte disparité dans les pratiques des lycéens. Par exemple, 41,9\% passent moins d'une demiheure par jour sur des réseaux sociaux alors que 20,3\% vont y consacrer plus de deux heures.

\begin{tabular}{|c|c|c|c|c|}
\hline $\begin{array}{l}\text { "En dehors du week-end et des va- } \\
\text { cances, combien de temps passez-vous, } \\
\text { en moyenne, chaque jour à : }\end{array}$ & $\begin{array}{l}\text { Moy. } \\
\text { (h/jour) }\end{array}$ & $\begin{array}{l}\text { Écart- } \\
\text { type } \\
\text { (h/jour) }\end{array}$ & $\begin{array}{l}\text { Moins } \\
\text { de } 30 \\
\mathrm{mn} / \text { jour }\end{array}$ & $\begin{array}{l}\text { plus de } \\
2 \mathrm{~h} / \text { jour }\end{array}$ \\
\hline $\begin{array}{l}\text { communiquer sur des réseaux so- } \\
\text { ciaux (Facebook, MSN, etc.)? }\end{array}$ & $1 \mathrm{~h}$ & 1,34 & $41,9 \%$ & $20,3 \%$ \\
\hline communiquer par SMS ? & $2 \mathrm{~h} 25$ & 1,86 & $20,6 \%$ & $52,7 \%$ \\
\hline $\begin{array}{l}\text { regarder des vidéos ou écouter de la } \\
\text { musique sur Internet? }\end{array}$ & $1 \mathrm{~h} 05$ & 1,38 & $8 \%$ & $25,6 \%$ \\
\hline $\begin{array}{c}\text { jouer sur ordinateur, un téléphone } \\
\text { ou une console? }\end{array}$ & $1 \mathrm{~h} 05$ & 1,42 & $57,1 \%$ & $19,4 \%$ \\
\hline
\end{tabular}

\footnotetext{
${ }^{3}$ De manière à pouvoir comparer le temps de travail selon certaines caractéristiques sociales et scolaires des lycéens, les échelles ordinales des deux variables précédentes ont été transformées en échelles de rapport en considérant le centre de chaque classe comme valeur de la distribution. Pour établir la durée moyenne hebdomadaire, le temps quotidien (du lundi au vendredi) a été multiplié par cinq et le temps du week-end par deux.

${ }^{4}$ Ces durées moyennes sont à relativiser dans la mesure où elles sont estimées sur la base d'une déclaration des lycéens qui, pour certains d'entre eux, ont pu rencontrer certaines difficultés à indiquer le temps véritablement consacré à certaines activités, notamment l'envoi de SMS. Par ailleurs, il serait erroné de sommer les durées de chaque activité puisque une partie des lycéens sont «multitâches » et effectuent en parallèle plusieurs activités.
} 


\begin{tabular}{|c|c|c|c|c|}
\hline $\begin{array}{c}\text { chercher des informations avec un } \\
\text { moteur de recherche (Google...) ? }\end{array}$ & 0 h47 & 0,94 & $55,8 \%$ & $9,1 \%$ \\
\hline
\end{tabular}

Tableau 2 • Temps quotidien consacré aux activités numériques

L'utilisation d'autres outils numériques est moins fréquente. La majorité des lycéens ne gèrent pas de blog, ne consultent pas de forums, ne créent pas de supports multimédia ou ne lisent pas la presse sur Internet. Ils seront par contre plus nombreux à utiliser des logiciels de bureautique et à communiquer par mail.

\begin{tabular}{|c|c|c|c|c|c|c|}
\hline $\begin{array}{l}\text { "Depuis la rentrée scolaire de } \\
\text { septembre, à quelle fréquence } \\
\text { avez-vous: }\end{array}$ & 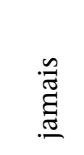 & 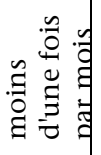 & 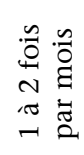 & 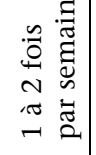 & 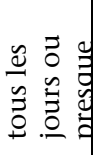 & 㐫 \\
\hline $\begin{array}{l}\text { utilisé des logiciels de bureau- } \\
\text { tique (Word, Open office...)? }\end{array}$ & 11,2 & 14,1 & 34,2 & 31,9 & 8,7 & $\begin{array}{c}100 \\
\%\end{array}$ \\
\hline communiqué par mail? & 32,9 & 22,2 & 22,7 & 16,3 & 5,9 & $\begin{array}{c}100 \\
\%\end{array}$ \\
\hline lu la presse sur Internet? & 50,2 & 17,6 & 14,6 & 11,5 & 6,2 & $\begin{array}{c}100 \\
\%\end{array}$ \\
\hline $\begin{array}{l}\text { programmé ou créé des sup- } \\
\text { ports multimédia (musique, } \\
\text { images, vidéos, etc.) ? }\end{array}$ & 53,0 & 14,0 & 15,2 & 12,7 & 5,2 & $\begin{array}{c}100 \\
\%\end{array}$ \\
\hline $\begin{array}{l}\text { participé ou consulté } \\
\text { des forums sur Internet? }\end{array}$ & 59,1 & 15,7 & 13,6 & 7,8 & 3,8 & $\begin{array}{c}100 \\
\%\end{array}$ \\
\hline $\begin{array}{l}\text { géré un blog ou un site Inter- } \\
\text { net? }\end{array}$ & 79,6 & 4,4 & 3,6 & 5,3 & 7,2 & $\begin{array}{c}100 \\
\%\end{array}$ \\
\hline
\end{tabular}

Tableau 3 - Fréquence des activités numériques

À partir des items des deux tableaux précédents (2 et 3), il est possible de dégager cinq profils d'utilisateurs du numérique grâce à une classification automatique en nuées dynamiques.

\begin{tabular}{|l|l|c|}
\hline & Durée moyenne quotidienne & $\begin{array}{c}\text { Fréquence moyenne } \\
\text { d'utilisation (sur une échelle } \\
\text { de 1 (jamais) à 5 (tous les } \\
\text { jours)) }\end{array}$ \\
\hline
\end{tabular}




\begin{tabular}{|c|c|c|c|c|c|c|c|c|c|c|c|c|}
\hline Classes & $\%$ & 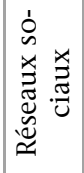 & $\sum_{n}^{\infty}$ & 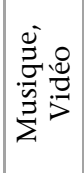 & $\stackrel{x}{\Xi}$ & $\begin{array}{l}\frac{0}{80} \\
8 \\
0 \\
0\end{array}$ & $\overline{\bar{\Xi}}$ & $\frac{60}{0}$ & 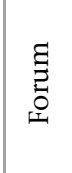 & $\begin{array}{l}\mathscr{\varpi} \\
\stackrel{\Xi}{\Xi}\end{array}$ & 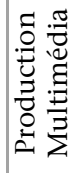 & 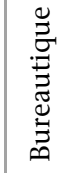 \\
\hline « Déconnectés » & $29,4 \%$ & Oh37 & Oh57 & Oh53 & Oh48 & Oh31 & 1,87 & 1,25 & 1,44 & 1,53 & 1,43 & 2,74 \\
\hline «Smistes » & $24,6 \%$ & $1 \mathrm{~h} 20$ & $4 \mathrm{~h} 17$ & $1 \mathrm{~h} 08$ & Oh27 & Oh38 & 2,11 & 1,33 & 1,44 & 1,60 & 1,71 & 3,00 \\
\hline «Ludiques» & $11,8 \%$ & $2 \mathrm{~h} 53$ & $4 \mathrm{~h} 01$ & $3 \mathrm{~h} 39$ & $3 \mathrm{~h} 30$ & $1 \mathrm{~h} 28$ & 1,96 & 1,66 & 1,93 & 1,78 & 2,13 & 2,85 \\
\hline $\begin{array}{l}\text { «Hyper- } \\
\text { connectés » }\end{array}$ & $10,8 \%$ & $2 \mathrm{~h} 03$ & $4 \mathrm{~h} 11$ & $2 \mathrm{~h} 04$ & Oh48 & $1 \mathrm{~h} 12$ & 2,99 & 3,21 & 2,41 & 2,85 & 3,66 & 3,63 \\
\hline "Académiques" & $23,4 \%$ & Oh31 & $1 \mathrm{~h} 03$ & Oh49 & Oh42 & Oh39 & 3,32 & 1,37 & 2,36 & 2,98 & 2,32 & 3,6 \\
\hline
\end{tabular}

Tableau 4 • Profils d'utilisateurs du numérique

Un premier profil s'oppose à tous les autres et rassemble les élèves qui utilisent très peu le numérique, les « déconnectés ». Cette classe ne présente pas de caractéristiques particulières. Une deuxième figure, les "smistes ", se singularise surtout par le temps extrêmement élevé à envoyer des SMS. On trouve dans cette classe significativement plus de filles $(71,8 \%)^{5}$, de lycéen(e)s professionnel(le)s et d'élèves hébergés en Internat. Une troisième classe, les «ludiques », rassemble surtout de forts consommateurs d'Internet qu'ils utilisent fréquemment pour communiquer, jouer, écouter de la musique ou visionner des vidéos. Les garçons et les lycéens professionnels sont davantage représentés dans cette classe. La classe des "hypercommunicants " présente certaines similitudes avec la classe des «ludiques». Ils consomment beaucoup de numérique pour un usage récréatif mais aussi pour s'informer ou produire des ressources. Les filles y sont davantage représentées. La dernière catégorie, les «académiques », utilise surtout le numérique pour s'informer et produire, mais très peu pour se divertir. Il donne le sentiment d'employer les outils numériques à des fins scolaires. Cette classe présente les caractéristiques des «bons élèves » (Daverne \& Dutercq, 2013) : note au brevet supérieure à la moyenne des autres élèves, mère plus fréquemment diplômée du supérieur, inscrit dans une filière générale.

\footnotetext{
${ }^{5}$ Cette mesure confirme une tendance déjà mise à jour par O. Martin (Martin, 2007).
} 


\begin{tabular}{|c|c|c|c|c|c|c|c|c|}
\hline Classe & 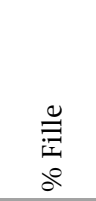 & 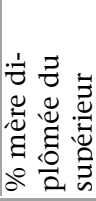 & $\begin{array}{l}\underset{\Xi}{\Xi} \\
\stackrel{\Xi}{\Xi} \\
\partial^{0}\end{array}$ & 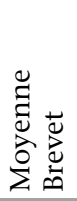 & 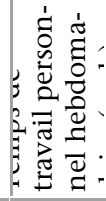 & 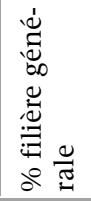 & 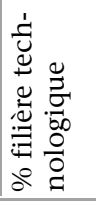 & 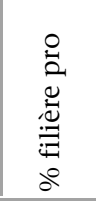 \\
\hline «Déconnectés» & $44,5 \%$ & $32,6 \%$ & $13,6 \%$ & 13,1 & 7h01 & $51,5 \%$ & $20,8 \%$ & $27,7 \%$ \\
\hline «Smistes» & $71,8 \%$ & $24,4 \%$ & $21,0 \%$ & 12,9 & $7 \mathrm{~h} 22$ & $39,0 \%$ & $23,8 \%$ & $37,2 \%$ \\
\hline « Ludiques» & $34,8 \%$ & $24,9 \%$ & $19,8 \%$ & 12,6 & $5 \mathrm{~h} 10$ & $31,9 \%$ & $19,2 \%$ & $48,9 \%$ \\
\hline $\begin{array}{c}\text { «Hyper- } \\
\text { communicants » }\end{array}$ & $69,8 \%$ & $27,2 \%$ & $16,2 \%$ & 12,7 & $6 \mathrm{~h} 27$ & $33,3 \%$ & $23,5 \%$ & $43,2 \%$ \\
\hline «Académiques» & $44,7 \%$ & $45,7 \%$ & $14,1 \%$ & 14,1 & $8 \mathrm{~h} 41$ & $65,9 \%$ & $21,1 \%$ & $13,0 \%$ \\
\hline Ensemble & $52,2 \%$ & $35,9 \%$ & $16,4 \%$ & 13,2 & 7h07 & $47,8 \%$ & $21,7 \%$ & $30,5 \%$ \\
\hline
\end{tabular}

Tableau 5 • Caractéristiques des profils d'utilisateurs du numérique

\subsection{Analyse multivariée du temps de travail personnel}

Pour estimer l'impact des usages numériques sur le travail hors classe des élèves, il convient de s'interroger préalablement sur les facteurs individuels et contextuels susceptibles d'expliquer la disparité du temps de travail personnel. Ce préalable apparait d'autant plus nécessaire que les usages numériques ne sont pas indépendants de ces différents facteurs, comme en attestent les profils d'utilisateurs présentés précédemment. Par exemple, un simple croisement entre le temps de travail et la fréquence de lecture de la presse sur Internet montre que ceux qui ne lisent jamais la presse travaillent en moyenne une heure de moins par semaine que les autres. Il serait alors tentant de conclure à un impact positif et significatif de la lecture sur le travail lycéen. Toutefois, on constate que les lycéens professionnels lisent moins que les lycéens généraux. Lorsque l'on contrôle la spécialité de formation des lycéens, l'impact de la lecture sur le temps de travail n'est plus significatif. Autrement dit, si les "grands » lecteurs travaillent davantage, c'est surtout parce qu'ils suivent des formations générales qui nécessitent un engagement plus conséquent dans les activités hors classe. Il importe donc de raisonner «toutes choses égales par ailleurs » pour évaluer le véritable impact du numérique. Les modèles réalisés ( $c f$. annexes) estiment progressivement l'effet des dimensions 
suivantes sur le temps de travail: les caractéristiques sociodémographiques; le diplôme préparé et la scolarité antérieure; les manières d'étudier ; les usages numériques.

\subsubsection{Influence des caractéristiques sociodémographiques}

Un premier modèle dans lequel figure uniquement le diplôme obtenu par chacun des deux parents et le sexe de l'élève explique 9,3\% de la variance. Les écarts sont significatifs dans ce premier modèle. D'une part, les lycéennes travaillent davantage que les lycéens; d'autre part, les élèves dont le père et la mère sont diplômés de l'enseignement supérieur travaillent environ deux heures de plus par semaine. Cela étant, cet écart associé au niveau d'études des parents ne devient plus significatif dans le deuxième modèle qui tient compte du diplôme préparé par les lycéens ${ }^{6}$. Ce résultat montre que cette forme d'engagement dans les études, évalué à travers le temps de travail, ne dépend pas du niveau social et culturel de la famille. Par contre, l'écart entre les filles et les garçons demeure très significatifs. Les lycéennes travaillent en moyenne $2 \mathrm{~h} 6 \mathrm{mn}$ de plus par semaine que les lycéens. L'écart s'explique surtout par des pratiques plus studieuses de la part des filles : 76\% d'entre elles travaillent tous les jours ou presque (versus 54,7\% pour les garçons), 52,7\% remettent leurs cours au propre (vs $28,7 \%$ ). Elles ne se contentent pas de relire les cours pour réviser. Elles réalisent plus fréquemment des fiches synthétiques (26,6\% vs 7,7\%). Elles vont fréquemment demander de l'aide en cas de difficultés, s'échanger des documents avec leurs camarades, compléter le cours en s'aidant des manuels scolaires et d'Internet. Les usages numériques sont également très clivés : les filles consacrent plus de temps aux textos et aux réseaux sociaux alors que les garçons joueront davantage.

\footnotetext{
${ }^{6}$ Un modèle dans lequel figurait la profession des parents à la place de leur niveau d'étude a été testé et conduit à la même conclusion. En raison des problèmes de multicolinéarité profession et niveau de diplôme sont fortement corrélés -, seul le niveau de diplôme a été finalement retenu pour conserver la stabilité du modèle.
} 


\begin{tabular}{|l|l|l|l|l|l|l|}
\hline \multicolumn{2}{|c|}{ Genre } & $\begin{array}{c}\text { Réseaux } \\
\text { sociaux }\end{array}$ & \multicolumn{1}{|c|}{ Textos } & $\begin{array}{c}\text { Vidéo } \\
\text { et/ou } \\
\text { musique }\end{array}$ & Jeu & $\begin{array}{c}\text { Moteur } \\
\text { de re- } \\
\text { cherche }\end{array}$ \\
\hline \multirow{2}{*}{ Masc. } & Moyenne & $\mathbf{1 h 0 6}$ & $\mathbf{1 h 5 8}$ & $\mathbf{1 h 2 9}$ & $\mathbf{1 h 3 8}$ & $\mathbf{0 h 4 9}$ \\
\cline { 2 - 7 } & Ecart-type & 1,30 & 1,76 & 1,38 & 1,56 & 1,00 \\
\hline \multirow{2}{*}{ Fém. } & Moyenne & $\mathbf{1 h 2 1}$ & $\mathbf{2 h 5 5}$ & $\mathbf{1 h 1 9}$ & $\mathbf{0 h 3 4}$ & $\mathbf{0 h 4 4}$ \\
\cline { 2 - 7 } & Ecart-type & 1,40 & 1,74 & 1,38 & 1,06 &, 90 \\
\hline \multirow{2}{*}{ Total } & Moyenne & $\mathbf{1 h 1 5}$ & $\mathbf{2 h 3 0}$ & $\mathbf{1 h 2 3}$ & $\mathbf{1 h 0 1}$ & $\mathbf{0 h 4 6}$ \\
\cline { 2 - 7 } & Ecart-type & 1,36 & 1,81 & 1,39 & 1,40 &, 94 \\
\hline \multicolumn{2}{|l}{ T de Student } & $-4,24^{\star * *}$ & $-11,65^{\star * *}$ & $2,66^{\star * *}$ & $17,66^{* * *}$ & $1,90^{*}$ \\
\hline
\end{tabular}

* écart peu significatif (seuil de 10\%); ** écart significatif (seuil de 5\%); ***écart très significatif (seuil de 1\%).

Tableau 6 • Temps consacré au numérique (en heures par jour) selon le sexe des élèves

Elles font un usage plus scolaire des outils numériques en employant plus fréquemment les logiciels de bureautique, en contactant leurs camarades pour résoudre un exercice ou préparer un devoir alors que les garçons privilégient les fonctionnalités ludiques du numérique (jeux, vidéo, musique). La dernière enquête PISA (OCDE, 2012) réalisée en 2009 portant sur 28 pays (hors France) révèle qu'en moyenne les garçons âgés de 15 ans utilisent significativement plus que les filles l'informatique à des fins ludiques. La plupart des études convergent vers ce constat (notamment Mercklé \& Octobre, 2012).

\subsubsection{Diplôme préparé, scolarité antérieure et régime scolaire.}

Le temps de travail dépend davantage de la spécialité que du niveau scolaire des lycéens.

Il existe une corrélation positive et significative du temps avec la moyenne obtenue au brevet, mais cette corrélation est peu élevée $(\mathrm{r}=0.26)$. Toutes choses égales par ailleurs, un lycéen qui a obtenu un point de plus au brevet travaille en moyenne 7 minutes de plus par semaine. C'est dire à quel point le niveau scolaire est peu explicatif de la variance $(6,7 \%)$ du temps de travail.

Ajoutons que le temps de travail n'est pas significativement différent que les lycéens soient en classe de seconde, de première ou de terminale.

Par contre, il existe une différence significative dans le temps de travail personnel entre les lycéens professionnels et les lycéens technologiques et 
généraux. Les premiers y consacrent $3 \mathrm{~h} 15 \mathrm{mn}$ de moins que les seconds. La spécialité de formation explique à elle seule près de $15 \%$ de la variance du temps. Selon Aziz Jellab (Jellab, 2009), peu d'enseignants de lycée professionnel exigent de devoirs à la maison parce qu'ils considèrent que les élèves ne les effectueront guère en raison de leur rapport distant aux savoirs scolaires et de leurs problèmes familiaux. Par ailleurs, ils travaillent moins régulièrement, demandent moins d'aide à leur entourage, utilisent moins les manuels scolaires, échangent rarement des documents avec leurs camarades et prennent davantage contact avec les enseignants pour des raisons personnelles que pour demander des explications sur une notion incomprise. Cette prise de distance avec le travail scolaire s'accompagne plus fréquemment de certains comportements interdits par l'institution : les lycéens professionnels envoient et/ou lisent souvent des textos durant les cours (22,5\% vs $10 \%$ pour les lycéens généraux) et consultent plus Internet pendant les enseignements.

Par ailleurs, les lycéens professionnels passent beaucoup plus de temps à envoyer des textos, à visionner des vidéos, à écouter de la musique, à jouer sur un ordinateur, à faire des recherches sur Internet et à consulter leur page personnelle sur les réseaux sociaux. Ce sont en définitive les plus gros consommateurs du numérique récréatif et communicatif. Par contre, ils lisent peu la presse sur Internet et utilisent rarement les logiciels bureautiques.

Enfin, les élèves hébergés en Internat ont des manières d'étudier et un rapport aux activités numériques singuliers. D'une part, ils déclarent travailler, toutes choses égales par ailleurs, $1 \mathrm{~h} 44 \mathrm{mn}$ de plus par semaine que les élèves externes ou demi-pensionnaires. Un surcroit de temps de travail personnel qui se concentre surtout du Lundi au Vendredi. Moins fréquemment absents, ils travaillent plus régulièrement et sollicitent davantage le personnel éducatif pour les devoirs. L'univers de l'internat, par les contraintes qu'impose l'hébergement en collectivité, induit des méthodes de travail particulières (Glasman, 2012) où la place du numérique est moins présente. Les internes ont effectivement moins l'occasion d'utiliser un ordinateur et Internet. Seuls $14,3 \%$ se servent d'un ordinateur tous les jours contre $27,5 \%$ pour les externes et demi-pensionnaires. Ils ne sont toutefois pas totalement « déconnectés » et vont surtout compenser cette privation par une intempérance du téléphone, y compris en classe où l'envoi de textos est fréquent. 


\subsubsection{Des manières d'étudier : entre activités chronophages et activités productives}

Les manières d'étudier des lycéens impactent fortement le temps de travail personnel. Certaines y sont significativement et positivement corrélées, telles que mettre sa prise de note au propre, apprendre par cœur ses cours ou réaliser des fiches synthétiques. À l'inverse, les lycéens qui se contentent de relire le cours ou laissent en l'état leur prise de notes, travaillent significativement moins. Ces différentes manières d'étudier illustrent des stratégies d'apprentissage en surface (e.g. recopier le cours) ou en profondeur (e.g. exercices et lectures complémentaires) qui font varier le temps de travail personnel.

L'environnement familial, amical et scolaire joue également un rôle important. Ceux qui communiquent avec leurs enseignants en dehors des cours et qui sollicitent leur entourage pour recevoir une aide dans la réalisation de leurs devoirs, travaillent davantage que les élèves isolés. Le recours à Internet pour réaliser les devoirs et les leçons est très fréquent. 73,2\% des lycéens consultent Internet le plus souvent pour compléter les cours et les manuels. Internet offre également l'opportunité de plagier des contenus ou récupérer des corrigés. 73,3\% des lycéens déclarent avoir déjà plagié tout ou partie des contenus sur des sites Internet et 38,6\% ont récupéré des corrigés sur des sites. Toutefois le plagiat reste occasionnel : seuls $15 \%$ déclarent recourir assez souvent ou très souvent au "copiercoller » et moins de 7\% récupèrent fréquemment des corrigés sur Internet (Michaut, 2013). Toutes choses égales par ailleurs, plus les lycéens usent du copier-coller, moins ils travaillent.

Les élèves privilégient-ils certaines méthodes en fonction de leur niveau scolaire et du temps de travail ? Une classification automatique en nuées dynamiques permet d'établir quatre figures de lycéens selon leur moyenne au brevet et le temps de travail personnel. Une première classe, «les productifs", est constituée des lycéens les plus studieux : ils travaillent près de deux fois plus que la moyenne des lycéens, utilisent souvent les manuels et communiquent fréquemment avec les enseignants en dehors des cours. Davantage de filles, de lycéens provenant de milieux favorisés, inscrits dans une filière générale et de bon niveau scolaire initial sont présents dans cette classe. Une deuxième catégorie, les «laborieux », est sur certains aspects proche de la précédente : ils utilisent les mêmes méthodes de travail mais sollicitent moins les enseignants. Ils se différencient toutefois sur le plan des caractéristiques sociales et scolaires. Les 
lycéens de milieux modestes et en formation technologique y sont relativement plus représentés. Ils ont également obtenu une moyenne au brevet significativement inférieure (-2.5 points) aux «productifs ». Tout laisse à penser que malgré un travail conséquent et un respect des règles scolaires, ils n'arrivent pas à produire des résultats à la hauteur de leur engagement. C'est tout l'inverse avec la figure des "dilettantes», d'anciens «bons " collégiens, plutôt de milieux intermédiaire ou favorisés, ayant rejoint la voie générale. Ils travaillent moins de cinq heures par semaine, sans "perdre » de temps à rédiger des fiches ou à réécrire leurs cours. La dernière catégorie, "les oisifs ", rassemble les élèves qui travaillent très peu et semblent les plus éloignés de la forme scolaire : ils communiquent rarement avec les enseignants et utilisent moins fréquemment les manuels scolaires. Provenant le plus souvent de milieux défavorisés, massivement inscrits dans les filières professionnelles, ils ont connu par le passé des difficultés scolaires.

\begin{tabular}{|l|c|c|c|c|c|}
\hline Classes & «Productifs » & «Laborieux & «Dilettantes » & "Oisifs » & Ensemble \\
\hline \% & $20,7 \%$ & $23,5 \%$ & $29,5 \%$ & $26,3 \%$ & $100 \%$ \\
\hline $\begin{array}{l}\text { Moyenne } \\
\text { Brevet }\end{array}$ & $\mathbf{1 4 , 6}$ & $\mathbf{1 2 , 1}$ & $\mathbf{1 4 , 7}$ & $\mathbf{1 1 , 2}$ & 13,2 \\
\hline $\begin{array}{l}\text { Temps de } \\
\text { travail (en } \\
\text { h/sem) }\end{array}$ & $\mathbf{1 3 h 5 4}$ & $\mathbf{9 h 2 4}$ & $\mathbf{4 h 3 5}$ & $\mathbf{2 h 4 7}$ & $7 \mathrm{~h} 05$ \\
\hline $\begin{array}{l}\text { Met ses cours } \\
\text { au propre }\end{array}$ & $47 \%$ & $\mathbf{5 1 \%}$ & $\mathbf{3 2 \%}$ & $38 \%$ & $41 \%$ \\
\hline $\begin{array}{l}\text { Rédige des } \\
\text { fiches }\end{array}$ & $\mathbf{2 2 \%}$ & $\mathbf{2 3 \%}$ & $\mathbf{1 3 \%}$ & $\mathbf{1 1 \%}$ & $16 \%$ \\
\hline $\begin{array}{l}\text { Utilise les } \\
\text { manuels }\end{array}$ & $\mathbf{7 2 \%}$ & $\mathbf{6 3 \%}$ & $54 \%$ & $\mathbf{3 5 \%}$ & $55 \%$ \\
\hline $\begin{array}{l}\text { Communique } \\
\text { avec les en- } \\
\text { seignants }\end{array}$ & $\mathbf{4 1 \%}$ & $21 \%$ & $20 \%$ & $\mathbf{1 6 \%}$ & $24 \%$ \\
\hline \% Fille & $\mathbf{6 4 \%}$ & $\mathbf{6 5 \%}$ & $\mathbf{3 8 \%}$ & $47 \%$ & $52 \%$ \\
\hline $\begin{array}{l}\text { \% mère di- } \\
\text { plômée du } \\
\text { supérieur }\end{array}$ & $\mathbf{5 0 \%}$ & $\mathbf{2 5 \%}$ & $\mathbf{3 9 \%}$ & $\mathbf{1 7 \%}$ & $32 \%$ \\
\hline $\begin{array}{l}\text { \% filière } \\
\text { générale }\end{array}$ & $\mathbf{7 0 \%}$ & $40 \%$ & $\mathbf{6 7 \%}$ & $\mathbf{1 5 \%}$ & $48 \%$ \\
\hline $\begin{array}{l}\text { \% filière } \\
\text { technologique }\end{array}$ & $24 \%$ & $\mathbf{3 4 \%}$ & $\mathbf{1 5 \%}$ & $\mathbf{1 6 \%}$ & $22 \%$ \\
\hline \% filière pro & $\mathbf{6 \%}$ & $\mathbf{2 6 \%}$ & $\mathbf{1 8 \%}$ & $\mathbf{6 9 \%}$ & $30 \%$ \\
\hline
\end{tabular}

En gras : les modalités significativement différentes au seuil de 5\%

Tableau 7 • Typologie des manières d'étudier 


\subsubsection{Usages numériques}

Le temps consacré à certaines activités numériques est négativement corrélé au temps de travail personnel : temps passé à envoyer des textos, sur les réseaux sociaux et les forums, temps consacré à la gestion d'un blog et le temps de jeu sur console ou sur Internet. À l'inverse, la recherche d'informations avec un moteur de recherche et l'utilisation de logiciels de bureautique augmentent significativement le temps de travail personnel. Enfin, le temps passé à visionner des vidéos ou à écouter de la musique sur Internet, à communiquer par mail, à lire la presse sur Internet ou à créer des supports multimédia n'affecte pas significativement le temps consacré aux devoirs et aux leçons.

En reprenant la typologie précédente, il est possible d'examiner les relations entre les manières d'étudier des lycéens et leurs usages numériques. Les "productifs» consomment relativement peu de numérique ludique et utilisent par contre significativement plus les logiciels de bureautique (y compris le courriel) et lisent davantage la presse sur Internet.Les « oisifs » présentent un profil totalement opposé : ils consacrent deux fois plus de temps aux réseaux sociaux, envoient davantage de textos, jouent plus, regardent fréquemment des vidéos sur Internet, gèrent régulièrement leur blog mais délaissent la bureautique et la presse. Les «laborieux » et les « dilettantes» sont dans une situation intermédiaire.

\begin{tabular}{|c|c|c|c|c|c|c|}
\hline & Classes & $\begin{array}{l}\text { "Produc- } \\
\text { tifs » }\end{array}$ & $\begin{array}{l}\text { «Labo- } \\
\text { rieux }\end{array}$ & $\begin{array}{l}\text { « Dilet- } \\
\text { tantes » }\end{array}$ & " Oisifs » & $\begin{array}{l}\text { En- } \\
\text { semble }\end{array}$ \\
\hline & $\%$ & $20,7 \%$ & $23,5 \%$ & $29,5 \%$ & $26,3 \%$ & $100 \%$ \\
\hline \multirow{5}{*}{ 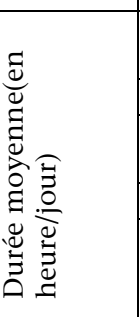 } & $\begin{array}{l}\text { Réseaux } \\
\text { sociaux }\end{array}$ & Oh48 & 1h12 & $1 \mathrm{ho3}$ & $1 h 38$ & 1h11 \\
\hline & SMS & $2 \mathrm{h03}$ & $2 h 38$ & $2 \mathrm{h04}$ & $2 h 55$ & $2 \mathrm{~h} 25$ \\
\hline & $\begin{array}{l}\text { Musique, } \\
\text { Vidéo }\end{array}$ & 1h08 & $1 \mathrm{~h} 21$ & 1h18 & $1 \mathrm{~h} 43$ & $1 \mathrm{~h} 23$ \\
\hline & Jeux & Oh36 & Oh54 & $1 \mathrm{~h} 06$ & 1h30 & $1 \mathrm{~h} 00$ \\
\hline & $\begin{array}{l}\text { Moteurs } \\
\text { de re- } \\
\text { cherche }\end{array}$ & Oh42 & Oh48 & 0h36 & Oh48 & Oh42 \\
\hline \multirow{5}{*}{ 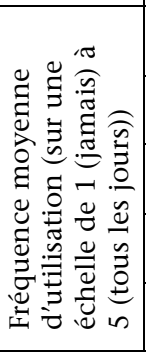 } & Mail & 2.8 & 2.3 & 2.5 & 1.9 & 2.4 \\
\hline & Blog & 1.3 & 1.4 & 1.4 & 1.9 & 1.5 \\
\hline & Forum & 1.7 & 1.6 & 2.0 & 1.8 & 1.5 \\
\hline & Presse & 2.2 & 1.9 & 2.1 & 1.8 & 2.0 \\
\hline & $\begin{array}{l}\text { Produc- } \\
\text { tion }\end{array}$ & 2.0 & 1.9 & 1.9 & 2.1 & 2.0 \\
\hline
\end{tabular}




\begin{tabular}{|l|l|l|l|l|l|l|}
\hline & $\begin{array}{l}\text { Multi- } \\
\text { média }\end{array}$ & & & & & \\
\cline { 2 - 6 } & $\begin{array}{l}\text { Bureau- } \\
\text { tique }\end{array}$ & $\mathbf{3 . 4}$ & 3.0 & 3.0 & $\mathbf{2 . 8}$ & 3.1 \\
\hline
\end{tabular}

En gras : les modalités significativement différente au seuil de 5\%

Tableau 8 • Manières d'étudier et usages numériques

\section{Conclusion}

Le traitement des données met en évidence l'articulation entre temps passé à des occupations à caractère numérique, caractéristiques sociodémographiques, scolarité et manières de réaliser son travail scolaire en dehors des cours. Les lycéens consacrent un temps beaucoup plus conséquent à des activités numériques qu'à leur travail personnel, hors de la classe. Ils sont globalement de forts consommateurs des outils numériques. Mais cette forte consommation a-t-elle une influence sur le travail personnel du lycéen ? À cette interrogation initiale, nous montrons qu'il existe bien une corrélation entre temps consacré à des activités numériques et temps de travail personnel. On doit cependant noter de fortes différences entre les usages récréatifs qui sont négativement corrélés au temps de travail et la recherche documentaire sur internet ou l'usage de logiciels de bureautique qui y sont positivement corrélés. Les pratiques numériques «ludiques » et «productives et informationnelles » opposent deux figures de lycéens : les " oisifs », dont le temps de travail est le plus faible, consacrent un temps important à échanger avec leurs pairs sur internet ou par SMS, à jouer ou à flâner sur internet. Les "productifs ", dont le temps de travail est élevé, pratiquent quant à eux plus volontiers la lecture électronique et utilisent deux fois moins les réseaux sociaux.

Le temps consacré à des pratiques numériques n'est pour autant ni la seule variable explicative, ni la plus significative. Le temps de travail scolaire hors de la classe est ainsi moins affecté par les pratiques numériques que par le diplôme préparé par les lycéens. Le temps de travail « hors de la classe » est ainsi fortement dépendant de la spécialité préparée par les jeunes. Les lycéens professionnels consacrent en effet moins de temps de travail personnel que les bacheliers technologiques et généraux. Les stratégies et les lieux d'apprentissage se révèlent également plus déterminants. Enfin, les lycéennes, plus studieuses comme le montre la littérature, travaillent plus que les garçons. Au final, les pratiques numé- 
riques n'expliquent que $4 \%$ de la variance supplémentaire du temps de travail personnel.

Si la méthode employée dans cette recherche a été fructueuse, elle nécessite cependant d'en poser au moins deux limites. La première est relative à la mesure du temps qu'il est difficile pour les lycéens d'estimer avec exactitude. Leur temps personnel est en effet fragmenté en de nombreuses activités enchevêtrées, difficilement dissociables, comme faire un travail scolaire tout en répondant à un texto ou en téléchargeant un film sur internet. De surcroit, le temps est difficile à quantifier. Il est par exemple compliqué pour un lycéen de déterminer le temps qu'il passe à envoyer ou recevoir des SMS. La seconde limite tient aux usages de dispositifs techniques qui entrainent une relative porosité entre la nature récréative ou scolaire des pratiques numériques. Par exemple, l'utilisation de Facebook dans le cadre du travail personnel permet d'allier sociabilité numérique et activité scolaire ; l'emploi d'un moteur de recherche permet tout à la fois de télécharger des vidéos et de se documenter pour préparer un exposé.

Par ailleurs, notre étude révèle une corrélation faible entre temps de travail et réussite au brevet. Ce résultat va dans le sens des comparaisons internationales qui montrent que le surcroît de travail n'augmente que très peu les performances scolaires (Meuret \& Bonnard, 2010).

Il conviendrait probablement de s'interroger sur les effets de certaines pratiques numériques sur les résultats scolaires et les compétences informationnelles car, comme le souligne l'enquête PISA (OCDE, 2012), il existe chez les jeunes de 15 ans un lien entre les usages modérés de l'internet (communication et recherche documentaire) et les compétences à naviguer sur internet et à comprendre des écrits numériques.

\section{Bibliographie}

Barrère A. (1997). Les lycéens au travail : tâches objectives, épreuves subjectives. Paris, PUF, $268 \mathrm{p}$.

Barrère A. (2011). L'Éducation buissonnière. Quand les adolescents se forment par eux-mêmes, Paris, Armand Colin, 228 p.

Bovill M., Livingstone S.-M. (2001). Bedroom culture and the privatization of media use. In Sonia-M. Livingstone, Moira. Bovill (Éd.), Children and their changing media environment: a European comparative study. Mahwah, N.J., USA, Lawrence Erlbaum Associates, p. 179-200. 
Breton P., Proulx S. (1989). L'explosion de la communication : la naissance d'une nouvelle idéologie. Paris-Montréal, La Découverte, Boréal, 282 p.

Carroll J.-B. (1963). A model of school learning. Teachers College Record, vol.64 $\mathrm{n}^{\circ} 8$, p.723-733.

Crahay M. (2012). L'Ecole peut-elle être juste et efficace de l'égalite des chances a l'égalite des acquis (2e édition.). Bruxelles, De Boeck, 460 p.

Cuban L. (1986). Teachers and machines: the classroom use of technology since 1920. New York, Teachers College Press, 152 p.

Daverne C., Dutercq Y. (2013). Les bons élèves. Paris, PUF, 232 p.

Delaunay-Téterel H., Le Douarin L. (2011). Le "net scolaire » à l'épreuve du temps « libre » des lycéens. Revue Française de Socio-Économie, vol.8 n², p 103-121.

Dieuzeide H. (1982). Marchands et prophètes en technologie de l'éducation. In Actes du colloque : Les formes médiatisées de la communication éducative. École normale supérieure de Saint-Cloud, p. 78-82.

Donnat O. (2009). Les pratiques culturelles des français à l'ère numérique : Enquête 2008. Paris, Editions La Découverte, 282 p.

Garrigues P., Galland O. (1989). La vie quotidienne des jeunes, du lycée au mariage. Economie et statistique, vol. $223 n^{\circ} 1$, p 15-23.

Glasman D. (2012). L'internat scolaire: Travail, cadre, construction de soi. Rennes, PU Rennes, 258 p.

Glevarec H. (2010). Les trois âges de la «culture de la chambre». Ethnologie française, vol.40 $n^{\circ} 1$, p 19-30.

GRISAY A. (1997). Evolution des acquis cognitifs et socio-affectifs des élèves au cours des années de collège. Disponible sur internet : ftp://trf.education.gouv.fr/pub/edutel/dpd/ni9726.pdf. (consulté le 10 juin 2013).

Jellab A. (2009). Sociologie du lycée professionnel : L'expérience des élèves et des enseignants dans une institution en mutation. Toulouse, Presses Universitaires du Mirail, $332 \mathrm{p}$.

Jouët J. (2000). Retour critique sur la sociologie des usages. Réseaux, vol.18 n¹00, p 487-521.

Jouët J. (2011). Des usages de la télématique aux Internet Studies. In Communiquer à l'ère numérique: regards croisés sur la sociologie des usages. Paris, France: Transvalor-Presses des Mines, p 45-90.

Martin O. (2007). La conquête des outils électroniques de l'individualisation chez les 12-22 ans. Réseaux, vol. 6 n 145-146, p. 335-366.

Mattelart A. (1994). L'invention de la communication. Paris, La Découverte, 400 p.

Mattelart A., Mattelart M. (2004). Histoire des théories de la communication. Paris, La Découverte, $123 \mathrm{p}$.

Mercklé P., Octobre S. (2012). La stratification sociale des pratiques numériques des adolescents. RESET - Recherches en Sciences Sociales sur Internet, vol $1 \mathrm{n}^{\circ} 1$. Disponible sur internet : http://www.journalreset.org/index.php/RESET/article/view/3 (Consulté le 20 juin 2013). 
Meuret D., Bonnard C. (2010). Travail des élèves et performance scolaire. Revue

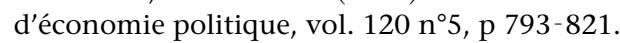

Michaut C. (2013). Les nouveaux outils de la tricherie scolaire au lycée, Recherches en éducation, vol. 16, p 131-142.

OCDE (2012). Résultats du PISA 2009 : Élèves en ligne - Technologies numériques et performance. Organisation de coopération et de développement économiques (OCDE), $424 \mathrm{p}$

OCLC (2005). College Students' Perceptions: Libraries \& Information Resources. Disponible sur internet : http://www.oclc.org/reports/perceptionscollege.en.html (consulté le 9 mai 2013).

Pasquier D. (2005). Cultures lycéennes: La tyrannie de la majorité. Paris, Autrement, $180 \mathrm{p}$.

Perrot J. (1984). Les influences des caractéristiques de l'offre d'éducation dans l'organisation du temps des élèves. Revue française de pédagogie, vol $69 \mathrm{n}^{\circ} 1, \mathrm{p}$ $35-48$.

Prensky M. (2001). Digital Natives, Digital Immigrants Part 1. On the Horizon, vol 9 issue 5, p 1-6.

Proulx S. (2004). L'irruption d'Internet dans les bibliothèques : un nouveau rapport au savoir? In Jean-Paul Baillargeon (Dir.), Bibliothèques publiques et transmission de la culture à l'orée du XXIe siècle, p. 61-73.

Rayou P. (2009). Épreuves d'aujourd'hui et métier de demain. Education et sociétés, vol $1 \mathrm{n}^{\circ} 23$, p 5-11.

Suchaut B. (2009). L'organisation et l'utilisation du temps scolaire à l'école primaire : enjeux et effets sur les élèves. Conférence à l'initiative de la ville de CranGevrier (Haute-Savoie). Disponible sur internet : http://halshs.archivesouvertes.fr/halshs-00395539 (consulté le 10 juin 2013). 


\begin{tabular}{|c|c|c|c|c|c|}
\hline & & \multicolumn{4}{|c|}{ Modèles } \\
\hline Modalitís de ruflerence & Modalints actives & 1 & 2 & 3 & 4 \\
\hline \multicolumn{2}{|l|}{ Constante } & $7.7 \mathrm{1}^{\mathrm{tat}}$ & $6,37^{154}$ & $5222^{4+24}$ & $5.33^{6+4}$ \\
\hline \multicolumn{6}{|c|}{ Caractéristiques sociodemographiques } \\
\hline Fille & Garçon & $-2.42^{2+1}$ & $-2.47^{2 * 1+}$ & $-1.43^{2.10}$ & $-1.22^{2 * 1+}$ \\
\hline Pére non diplómé du supérieur & \multirow{2}{*}{\begin{tabular}{|l|} 
Père diplómé du supérieur \\
Mere diplómée du
\end{tabular}} & $0.99^{* 1+1}$ & $0.97 \mathrm{~ns}$ & $0.29 n s$ & $0.26 n s$ \\
\hline Mère non điplômée du supérieur & & $1.28^{* *}$ & $0.43 \mathrm{~ns}$ & $0.3 \mathrm{~ns}$ & $0.21 \mathrm{~ns}$ \\
\hline \multicolumn{6}{|c|}{ Diplóme préparé et scolarité antérieure } \\
\hline \multirow{4}{*}{ Bac gentral } & Bac technologique & & $-0.13 \mathrm{~ns}$ & $0.15 \mathrm{~ns}$ & $0.02 \mathrm{~ns}$ \\
\hline & \begin{tabular}{|l|} 
Bac professionnel \\
\end{tabular} & & $-3.79 \%$ & $-3,6 \%$ & $-2.72^{\cdots \cdots}$ \\
\hline & Première & & $0.66^{* *}$ & $0.44^{*}$ & $0.24 \mathrm{~ns}$ \\
\hline & Terminale & & $0.41 \mathrm{~ns}$ & $0.37 \mathrm{~ns}$ & $0.17 \mathrm{~ns}$ \\
\hline Externe ou demi-pensionnalire & \begin{tabular}{|l|l} 
Interne \\
\end{tabular} & & $2.85^{5+0}$ & $279^{6+0}$ & $2.77^{2+4}$ \\
\hline \multicolumn{2}{|c|}{ Moyenne génerade obtenue au brevet des colleges (sur zo) } & & $0.28^{8+4}$ & $0.43^{*+4}$ & $0.09 \mathrm{~ns}$ \\
\hline \multicolumn{6}{|c|}{ Manières d'étudier } \\
\hline \multirow[b]{2}{*}{ Laisse ses cours en rétat } & met ses cours au propre & & & $0,72^{24+4}$ & $0,65^{\circ+4}$ \\
\hline & $\begin{array}{lll}\text { tape ses cours sur } \\
\text { ordinateur }\end{array}$ & & & $1.37^{* * *}$ & $0.93^{* *}$ \\
\hline \multirow{2}{*}{$\begin{array}{l}\text { Révise gèneralement.. } \\
\text { - en relisant ses cours }\end{array}$} & en apprenant par corur & & & $1.01^{2 *+}$ & $1.00^{2 * 1+}$ \\
\hline & $\begin{array}{l}\text { en realisant des fiches } \\
\text { synthétiques }\end{array}$ & & & $0.80^{* \ldots+}$ & $0.77^{*+*}$ \\
\hline \multirow{5}{*}{$\begin{array}{l}\text { Réalise principalement ses } \\
\text { devoirs en utilisant : } \\
\text {-cours, manuels et Internet }\end{array}$} & atucuin support & & & $-2.99^{21+1}$ & $-2.60^{\circ+10}$ \\
\hline & uniquement le cours & & & 1.29 & $-207^{* *+1}$ \\
\hline & \begin{tabular}{|l|l} 
cours et manuels \\
\end{tabular} & & & $0.4 \mathrm{~ns}$ & $0.47^{\mathrm{ns}}$ \\
\hline & cours et Internet & & & $-1.04^{*+*}$ & $-0.95^{2 *+\cdots}$ \\
\hline & \begin{tabular}{|l|l} 
seulement Internet \\
\end{tabular} & & & $-2.49^{-20}$ & $-2.14^{2.00}$ \\
\hline $\begin{array}{l}\text { Ne communique pas avec les } \\
\text { enselgnants en dehoes des } \\
\text { cours }\end{array}$ & $\begin{array}{l}\text { communique avec les } \\
\text { enseignants en dehors des } \\
\text { cours }\end{array}$ & & & $1.35 \%$ & $43^{* \cdots}$ \\
\hline \multicolumn{2}{|c|}{$\begin{array}{l}\text { Fréquence de sollicitation de l'entourage pour aide aux devoirs } \\
\text { (de jamais a trés souvent) }\end{array}$} & & & $0.56 \cdots$ & $0.57^{* * *}$ \\
\hline \multicolumn{2}{|c|}{ Plagie des contenus sur Internet (de jamals $d$ trds souvent) } & & & $-0.28^{ \pm+4}$ & $-0.28^{2+4}$ \\
\hline \multicolumn{6}{|c|}{ Usages numeriques } \\
\hline \multicolumn{2}{|c|}{$\begin{array}{l}\text { Temps de communication quotidien sar des réseaux sociaux } \\
\text { (en heures) }\end{array}$} & & & & $-0.28^{* *+}$ \\
\hline \multicolumn{2}{|c|}{ Temps de communication quotidien pur SMS (en beures) } & & & & $-0.133^{4+4}$ \\
\hline \multicolumn{2}{|c|}{$\begin{array}{l}\text { Temps quotidien de visionnage de vidéos ou d'écoute de } \\
\text { musique sur Internet (en heures) }\end{array}$} & & & & $-0.02 \mathrm{~ns}$ \\
\hline \multicolumn{2}{|c|}{$\begin{array}{l}\text { Temps quotidien de jeu sur un ordinateur, un teléphone ou } \\
\text { une console (en heures) }\end{array}$} & & & & $-0.16^{* *}$ \\
\hline \multicolumn{2}{|c|}{$\begin{array}{l}\text { Temps quotidien de recherche dinformations avec un moteur } \\
\text { de recherche (en heures) }\end{array}$} & & & & $0.46^{* * *}$ \\
\hline \multicolumn{2}{|c|}{ 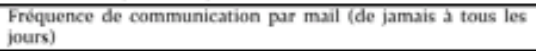 } & & & & o.n ns \\
\hline Frequence de gestion d'un blog (de & jamais a tous les jours) & & & & $-0.23^{* *}$ \\
\hline $\begin{array}{l}\text { Fréquence de communication sur c } \\
\text { les jours) }\end{array}$ & des forums (de jamais a tous & & & & $0.39^{* *+}$ \\
\hline $\begin{array}{l}\text { Fréquence de lecture de la pressh } \\
\text { tous les jours) }\end{array}$ & e sur lnternet (de jamais d & & & & o.or as \\
\hline $\begin{array}{l}\text { Fréquence de création de support } \\
\text { tous les jours) }\end{array}$ & is multimblia (de jamals d & & & & o.aq ns \\
\hline $\begin{array}{l}\text { Fréquence d'utilisation de logiciels } \\
\text { d tous les jours) }\end{array}$ & s de bureautique (de jamais & & & & $0.46^{* *}$ \\
\hline $\mathrm{R}^{2}$ : pourcentage de variance expliq & & $8.7 \%$ & $24.0 \%$ & $32.6 \%$ & $36.6 \%$ \\
\hline
\end{tabular}

Annexe 1 - Régression linéaire du temps de travail personnel en heures par semaine (hors enseignement)

Note de Lecture : les coefficients indiquent le nombre d'heures de travail personnel à ajouter ou à soustraire à la constante. Par exemple, dans le modèle 1, les lycéens déclarent consacrer en moyenne 2,64h de moins que les lycéennes. Le modèle 4 permet d'estimer, toutes choses égales par ailleurs, l'écart de temps 
selon le genre qui n'est plus que de 1,19h. Sont également indiqués les seuils critiques dont la lecture est la suivante : ns : écart non significatif entre la modalité active et la modalité de référence ; *écart peu significatif (seuil de 10\%);

** écart significatif (seuil de 5\%); ***écart très significatif (seuil de 1\%). 